\section{United Kingdom National Ophthalmology Database Study: Diabetic Retinopathy; Report 1: prevalence of centre-involving diabetic macular oedema and other grades of maculopathy and retinopathy in hospital eye services}

TDL Keenan ${ }^{1}$, RL Johnston ${ }^{2,3}$, PHJ Donachie ${ }^{2,3}$, JM Sparrow ${ }^{2,4}$, IM Stratton ${ }^{2,5}$ and P Scanlon ${ }^{3,5}$

\author{
${ }^{1}$ Department of \\ Ophthalmology, Manchester \\ Royal Eye Hospital and \\ University of Manchester, \\ Manchester, UK \\ ${ }^{2}$ The Royal College of \\ Ophthalmologists' National \\ Ophthalmology Database \\ project, London, UK

\section{${ }^{3}$ Department of Ophthalmology, Gloucestershire Hospitals NHS Foundation Trust, Cheltenham, UK}

\begin{abstract}
Aims To report estimates of the prevalence of diabetic retinopathy (DR) and maculopathy grades for a large cohort of patients managed by the UK hospital eye service (HES).

Methods Anonymised data were extracted from 30 UK NHS hospital trusts using a single ophthalmic electronic medical record (EMR) for the period from April 2000 to November 2010 to create the National Ophthalmology Database (NOD). From 2007, the EMR facilitated capture of a nationally agreed-upon standardised data set (DR Structured Assessment) relating to the presence or absence of clinical signs of DR and maculopathy. An algorithm in the software automatically calculated the Early Treatment of Diabetic Retinopathy Study grades of retinopathy and maculopathy. Results Between 2007 and 2010, 307538 patients had data on the NOD, with 76127 (24.8\%) patients having been recorded as having diabetes. The proportion of patients with diabetes who had a structured assessment increased from $50.7 \%$ (2007) to $86.8 \%$ (2010). In each NHS year, $12.6-20.6 \%$ of
\end{abstract}

eyes with structured assessments had no DR; 59.6-67.3\% had non-proliferative DR; and 18.3-20.9\% had active or regressed proliferative DR. Clinically significant macular oedema was present in $\mathbf{1 5 . 8 - 1 8 . 1 \%}$ of eyes, and in $8.7-10.0 \%$ of eyes, this involved the central macula.

Conclusion This study provides contemporary estimates of the prevalence of retinopathy and maculopathy grades in a large cohort of patients with diabetes managed by the UK HES. Centre-involving diabetic macular oedema, potentially amenable to antiVEGF therapy, is present in the eyes of almost $10 \%$ of these patients. This information is useful for clinicians, health-care economists, and commissioners involved in planning and delivering diabetic eye services.

Eye (2013) 27, 1397-1404; doi:10.1038/eye.2013.196; published online 20 September 2013

Keywords: diabetes mellitus; diabetic retinopathy; diabetic maculopathy; centre-involving diabetic macular oedema; clinically significant macular oedema; epidemiology
${ }^{4}$ Department of Ophthalmology, Bristol Eye Hospital and University of Bristol, Bristol, UK

${ }^{5}$ Department of Ophthalmology, Gloucestershire Diabetic Retinopathy Research Group, Gloucestershire Hospitals NHS Foundation Trust, Cheltenham, UK

Correspondence: RL Johnston, Department of Ophthalmology, Gloucestershire Hospitals NHS Foundation Trust, Cheltenham General Hospital, Sandford Road, Cheltenham GL53 7AN, UK. Tel: +08454 222529; Fax: +08454 222585 . E-mial: Rob.Johnston@ glos.nhs.uk

Received: 3 October 2012 Accepted in revised form: 20 July 2013 20 September 2013
Published online: 


\section{Introduction}

Diabetic retinopathy (DR) is the most common cause of visual loss in the working age population of the United Kingdom. ${ }^{1,2}$ The management of patients with DR forms a large and increasing proportion of hospital eye service (HES) activity in the United, ${ }^{3}$ and the prevalence of diabetes is predicted to continue increasing over the next decades, ${ }^{4}$ with a concomitant rise in DR.

The last decade has seen significant changes in the organisation of care for patients with DR, with the introduction of national screening programmes for DR in all four countries of the UK. ${ }^{2,3,5-8}$ However, little up-todate information is available on the number of patients seen for DR in the HES, the proportion with different grades of DR and maculopathy, the number undergoing laser photocoagulation or intravitreal therapy, and their clinical outcomes.

These data are required to establish national standards of care and to enable patients, ophthalmologists, and managers to benchmark their personal or local outcomes. They are also essential for the planning of health-care requirements in the future and for evidence-based commissioning of services. ${ }^{9}$ In particular, the prevalence of centre-involving diabetic macular oedema (CIDMO) in the UK HES has important implications for clinicians and commissioners, given the recent licensing ${ }^{10}$ and NICE approval ${ }^{11}$ of ranibizumab for this indication.

The NHS Connecting for Health's 'Do Once and Share' programme defined detailed data sets to be collected within electronic medical record (EMR) systems, including the following ophthalmology data sets: Diabetic Eye Disease (DED-DOAS), the Cataract National Data set, and the Glaucoma Data set. ${ }^{12}$ The Royal College of Ophthalmologists (RCOphth) is responsible for professional standards in the practice of ophthalmology for the benefit of the general public. ${ }^{13}$ The National Ophthalmology Database (NOD) ${ }^{14}$ has been created under its auspices to collate anonymised data collected as a by-product of routine clinical care using EMR. The database conforms to nationally agreedupon data standards, with the aim of providing highquality data for national audit, research, and revalidation of clinicians. This project builds on a series of studies where clinicians have pooled EMR data to update benchmark standards for cataract and vitreo-retinal surgery. ${ }^{15-19}$

\section{Aims}

The primary aim of this study was to report estimates of the prevalence of each grade of DR and maculopathy for a large cohort of patients managed by the UK HES.

\section{Materials and methods}

\section{Data extraction}

Data were extracted till 30 November 2010 from 30 NHS trusts across the UK that use a single EMR system (Medisoft Ophthalmology, Medisoft Limited, Leeds, UK). The lead clinician and Caldicott Guardian (who oversees data protection) at each centre gave written approval for the data extraction. This study was conducted in accordance with the declaration of Helsinki and the UK's Data Protection Act.

\section{DR structured assessments}

A 'DR structured assessment' is defined as the documentation of the presence or absence of a specified minimum number of clinical signs of DR and maculopathy for each eye. When all mandatory fields are completed, an algorithm in the EMR system automatically calculates the grade of DR and maculopathy according to the Early Treatment of Diabetic Retinopathy Study (ETDRS) International ${ }^{20}$ and NHS Diabetic Eye Screening Programme (DESP) ${ }^{21}$ classifications of DR. The EMR's DR structured assessment module was progressively implemented at sites from 2006 onwards, and its completion is usually made compulsory in medical retina clinics for patients with diabetes. Whether CIDMO was present can be calculated based on an analysis of data items recorded for each eye in the macula 'retinal thickening' field of the structured assessment. All structured assessment results for eyes recorded as 'ocular prosthesis' or for which 'other pathology makes DR grading impossible/not relevant' were excluded from analysis.

Estimates of the proportions of eyes with each grade of DR and maculopathy per NHS year utilised the last structured assessment record in that year for each eye. Results per year are displayed according to the NHS year, which runs from 1 April to 31 March. Data based on the diabetic status (diabetic, not diabetic, or not recorded) were available from the 2003 NHS year onwards, and data from structured assessments were available from the 2007 NHS year onwards, when the EMR system incorporated the algorithms defined in the DED-DOAS project. This analysis reports results for the patients for whom data were recorded on the NOD from the 2007 NHS year till the completion of the initial data extraction in November 2010. Analysis was performed using STATA version 11 (StataCorp LP, College Station, TX, USA). 


\section{Results}

Between 2007 and 2010, 307538 patients from 30 NHS hospital trusts have had data aggregated on the NOD: 138431 (45.0\%) were male; 168708 (54.9\%) were female; and sex was not specified for $399(0.1 \%)$. Ethnicity was not recorded for $194324(63.2 \%)$ patients. Among the 113214 patients for whom it was recorded, $90.6 \%$ were Caucasian, $4.3 \%$ were Black, $3.3 \%$ were Asian, $0.5 \%$ were of mixed race, and $1.3 \%$ belonged to other ethnicities. The median age at the time of the patient's first record on the NOD was 72 years (interquartile range: $59-80$ years; range: $0-110$ years). Diabetic status was not recorded for 131035 (42.6\%) patients; 76127 (24.8\%) patients were recorded as diabetic; and $100376(32.6 \%)$ patients were not diabetic. The median age of patients recorded as diabetic at the time of their first record on the NOD was 68 years (interquartile range: 57-77 years; range: 4-108 years).

The proportion of patients seen in the HES who were recorded as having diabetes remained stable at $30.1 \%$ in the 2007 NHS year and $31.8 \%$ in the last complete NHS year (2009). This proportion was higher for men than women (around 35\% versus 25\% in 2009, respectively), consistently with the higher prevalence of diabetes among men in the UK population. ${ }^{4}$

\section{DR structured assessments}

A total of 48570 eyes from 24292 patients with diabetes had one or more structured assessments recorded. Data on 120 eyes were excluded from analysis owing to the recording of 'other pathology makes DR grading not relevant/impossible' or 'presence of an ocular prosthesis', leaving 48450 eyes from 24280 patients with structured assessments eligible for analysis. The proportion of patients with diabetes who had a structured assessment recorded increased from $50.7 \%$ in 2007 to $86.8 \%$ in 2010, and the number of sites using the DR structured assessment module of the EMR increased from 3 in 2007 to 18 in 2010.

\section{ETDRS grades and CIDMO in the last structured assessment}

Of the 48450 eyes with structured assessment data at the time of their last record, 679 eyes had either DR or maculopathy that was not possible to grade. Of the remaining 47771 eyes, 11356 (23.8\%) eyes had no DR, 21986 (46.0\%) had mild non-proliferative DR (NPDR), 6963 (14.6\%) had moderate NPDR, 2380 (5.0\%) had severe NPDR, and $5086(10.6 \%)$ had proliferative DR (PDR).
Clinically significant macular oedema (CSMO) was present in 6664 (13.9\%) eyes, with $3116(6.5 \%)$ eyes having non-centre-involving CSMO and 3548 (7.4\%) having CIDMO. There were more eyes with CIDMO than with non-centre-involving CSMO among eyes with PDR, severe NPDR, and moderate NPDR (Figure 1).

\section{Proportions of eyes with DR and maculopathy by year}

During each year of the study period, the proportion of eyes per year with no DR, NPDR, and active or regressed PDR varied between $12.6 \%$ and $20.6 \%, 59.6 \%$ and $67.3 \%$, and $18.3 \%$ and $20.9 \%$, respectively (Figures 2 and 3 ).

The proportion of eyes per year with DR but no macular oedema, macular oedema but no CSMO, CSMO but not centre-involving, CIDMO, and eyes that could not be graded for diabetic maculopathy ranged from $57.3 \%$ to $64.8 \%, 3.6 \%$ to $4.0 \%, 7.0 \%$ to $8.2 \%, 8.7 \%$ to $10.0 \%$, and $0.6 \%$ to $1.2 \%$, respectively (Figure 4 ).

\section{Discussion}

This is the first major study in the UK to harness the benefits of EMR systems to collate and analyse data on the grades of DR, and in particular, to give an estimate of the prevalence of CIDMO in patients attending the HES. The data are likely to be representative of the whole UK HES as they were obtained from 30 NHS hospitals, 18 of which used the DR structured assessment module. The hospitals are widely scattered across the UK and serve populations that vary in terms of age structure, ethnic mix, prevalence of diabetes, and socio-economic

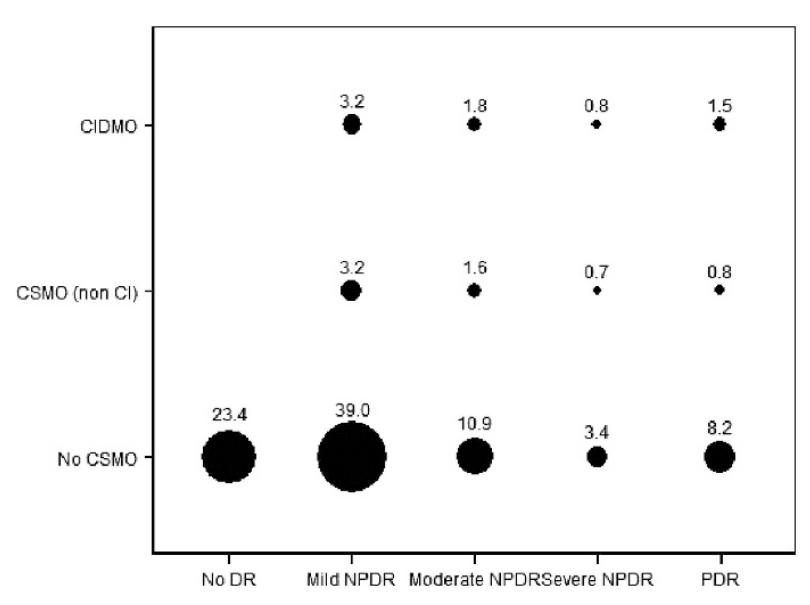

Figure 1 Grade of diabetic maculopathy versus grade of diabetic retinopathy for eyes at their most recent gradable DR Structured Assessment. Each circle represents the proportion of eyes with that combination of retinopathy and maculopathy grades. The labels above each circle display the proportions as percentages $(N=48450)$. 


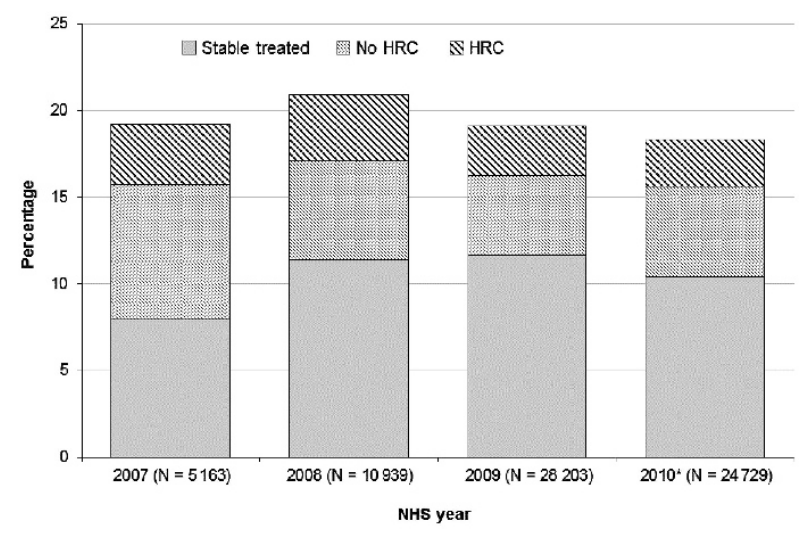

Figure 2 The proportion (\%) of eyes with each grade of PDR by NHS year, where HRC $=$ high-risk characteristics: $(N=$ the number of eyes with a gradable DR structured assessment each year). *The NHS year is from 1 April to 31 March; the 2010 NHS year is not complete; data are displayed up to 30 November 2010.

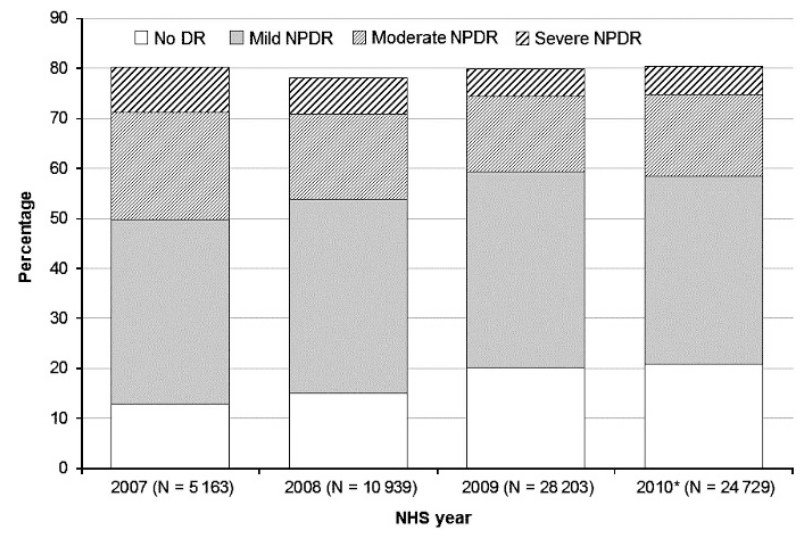

Figure 3 The proportion (\%) of eyes with each grade of NPDR by NHS year. ( $N=$ the number of eyes with a gradable DR structured assessment each year). *The NHS year is from 1 April to $31 \mathrm{March}$; the 2010 NHS year is not complete; data are displayed up to 30 November 2010.

deprivation. This is particularly useful, as heterogeneity may exist between different regions and countries within the UK, in terms of the proportions of people with different grades of DR and maculopathy seen in the HES. Potential reasons for this heterogeneity include differences in DR-screening protocols (for example, two fields in England versus one in Scotland, and variation in rates of mydriatic use), grading protocols, disease duration before diagnosis and screening, control of glycaemic and hypertensive factors, and screening inclusion/exclusion criteria..$^{5-8,22-24}$

However, it should be acknowledged that these data were obtained from one EMR supplier. This was because at the time of the data extraction, only one EMR supplier collected the nationally agreed-upon DED-DOAS data set.

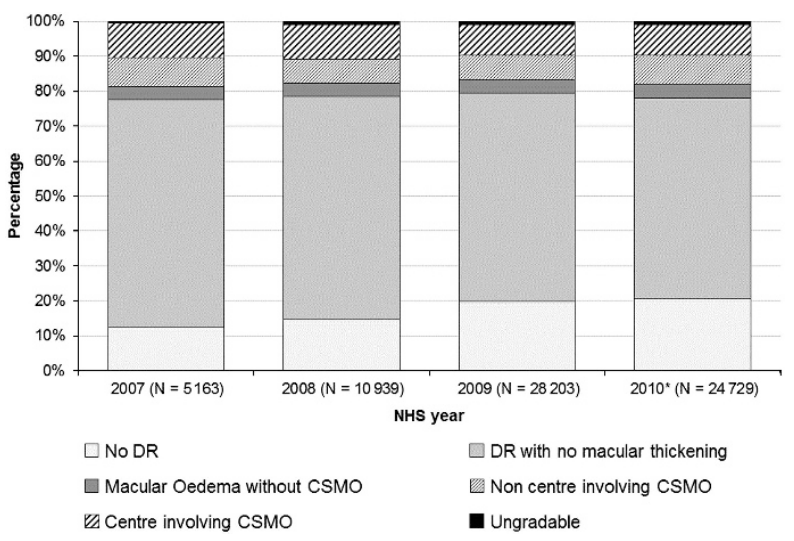

Figure 4 The proportion (\%) of eyes with each grade of diabetic maculopathy, by NHS year. $(N=$ the number of eyes with a DR structured assessment each year). *The NHS year is from the 1 April to 31 March; the 2010 NHS year is not complete; data are displayed up to 30 November 2010.

It is possible that units at the forefront of implementing EMR systems may not be typical of all units.

Furthermore, during the study period, the number of sites using the DR module increased from 3 to 18 , and the collated data do not allow us to make any comment on the proportion of doctors in each department that routinely used the EMR. However, the robustness of the data is aided by the prospective collection of a standardised data set as a by-product of routine clinical care, and by evidence that the proportion of patients with diabetes seen in hospital eye departments who had a structured DR assessment performed grew substantially during the study period from $51 \%$ in 2007 to $87 \%$ in 2010 . The study suggests that the management of patients with diabetes forms a significant component of ophthalmic activity in the UK, a situation that is likely to be mirrored in other developed countries.

The structured assessment of the presence or absence of the signs of DR conforming to the nationally agreedupon data set was made available within the EMR from 2007. Our data confirm that this facility is increasingly used when clinicians are assessing grades of DR and maculopathy, reflecting increased implementation, and usage of the module. Completion of this screening is usually made compulsory within medical retina clinics but is less frequently used when assessing patients with diabetes for ocular pathology unrelated to diabetes; hence, we would never expect completion of the structured assessment screening to approach $100 \%$. If all mandatory fields are completed, an algorithm within the EMR automatically calculates the grade of DR and maculopathy according to the ETDRS classification, ${ }^{20}$ ensuring standardisation of data collection. This allows a contemporary estimate of the prevalence of each grade of retinopathy and maculopathy in a large cohort of 
patients managed by the UK HES. Approximately $20 \%$ of eyes have no DR, $45 \%$ have mild NPDR, $14 \%$ have moderate NPDR, 5\% have severe NPDR, 6\% have active PDR, and $10 \%$ have stable-treated PDR. Almost $14 \%$ of eyes have CSMO and just over half of these eyes $(7.4 \%)$ have CIDMO. The ETDRS grade 61 (later 63, that is, photocoagulation scars together with fibrosis but no active neovascularisation) has a text description within the DR structured assessment of 'stable-treated PDR' and is equivalent to the new NHS DESP grade of R3s; this provides the first estimate of the proportion of eyes that may be safe to return to annual screening (depending on the status of the fellow eye and maculae).

No studies have been published in the recent literature, to our knowledge, of the prevalence of DR or maculopathy in the UK HES. Existing UK studies generally report prevalence estimates at the population level (that is, all individuals with DM) or at entry into DR-screening programmes. One previous study, the national DR laser treatment audit of 1995, did examine types of maculopathy treated in the UK HES, ${ }^{25}$ as well as features of PDR. ${ }^{26}$ However, the maculopathy grades reported are not in line with current classification systems, and the audit study preceded the advent of optical coherence tomography (OCT), which makes meaningful comparison with our data difficult. In addition, the case mix of patients with diabetes managed by the HES has probably changed substantially since 1995, with an increase in the number of patients with DR, the introduction of screening programmes, the advent of OCT, and changes in laser design and treatment thresholds. ${ }^{3}$

The absence of other UK HES studies makes comparison difficult. Prevalence data for DR and maculopathy at the population level have been reviewed previously for the $\mathrm{UK}^{27}$ and recently across the globe. ${ }^{28}$ For the UK, the prevalence of any grade of DR in the Caucasian diabetic population has been estimated to be between $17 \%$ and $41 \%$, and between $1 \%$ and $8 \%$ for PDR. ${ }^{29-35}$ Another UK study reported DR prevalence to be $36 \%$ among white Europeans versus $45 \%$ among South Asians. ${ }^{36}$ Worldwide, the prevalence of any grade of DR (among people with diabetes aged 20-79 years) has been estimated to be $35 \%$, and that of PDR to be $7 \% .37$

Again, no studies have been published, to our knowledge, of the prevalence of CIDMO either in the UK HES or in other Western countries. As above, existing UK studies have reported prevalence estimates for all diabetic macular oedema (DMO) and/or CSMO at the population level. The prevalence of DMO in the UK diabetic population was reported to be $6 \%$ among white Europeans and $12 \%$ among South Asians. ${ }^{36}$ For CSMO, the UK prevalence has been estimated to be $6 \%$ (in an inner city setting), though this study involved biomicroscopy, not OCT. ${ }^{35}$ Importantly, epidemiological models have suggested that the number of patients with DMO in the UK will increase in future from 188000 (2010) to 236000 (2020). ${ }^{38}$

Our study also provides estimates of the prevalence of CIDMO and CSMO for a given grade of DR among patients seen in the UK HES (see Figure 1). For example, $14 \%$ of eyes with mild NPDR, $24 \%$ of eyes with moderate NPDR, $31 \%$ of eyes with severe NPDR, and $22 \%$ of eyes with PDR also had CSMO (whether or not centre-involving). No previous studies have published similar data, though two studies have reported DMO prevalence figures by grade of DR. The large Wisconsin study in the USA reported in 1984 that the prevalence of DMO was 3\% (diabetes duration: 0-14 years) or $6 \%$ ( $\geq 15$ years) in mild NPDR, 37\% (0-14 years) or $63 \%$ ( $\geq 15$ years) in moderate/severe NPDR, and $73 \%$ (0-14 years) or $74 \%$ ( $\geq 15$ years) in PDR. ${ }^{39}$ The figures in our study are higher for mild NPDR (14\% versus 3-6\%) because individuals with mild NPDR at screening will only be referred to the UK HES in the presence of significant maculopathy. However, our figures for PDR (22\% versus 73-74\%) may be much lower because we are considering only CSMO, rather than all DMO, and perhaps because of better control of glycaemic and hypertensive factors, earlier treatment of PDR, and the impact of screening. More recently, a Japanese study also found a significant correlation between the severity of DMO and the DR grade. ${ }^{40}$ In their report, $28 \%$ of patients with mild/moderate NPDR, 67\% with severe NPDR, and 51\% with PDR also had DMO. However, some caution is required in attempting to compare figures between these reports: these studies were conducted in different countries at different periods, that is, with different genetic and environmental health determinants. Inclusion and exclusion criteria for the study populations were also different, as were technologies and drugs available for the detection and treatment of DR and maculopathy.

The information presented in this study provides an evidence base for commissioners, clinicians, and managers when planning appropriate allocation of workforce and resources for local and national diabetic eye services, as well as for the pharmaceutical industry. ${ }^{9}$ In particular, these data are useful for calculating the proportion of patients with diabetes seen in the HES who are likely to require pan-retinal photocoagulation, macular laser, and/or intravitreal anti-VEGF therapy, as well as diagnostic tests such as fundal photography, OCT imaging, and fluorescein angiography. ${ }^{3}$

It may be difficult to extrapolate our prevalence data on each grade of DR within the HES to the whole UK population with diabetes as there are few reliable data on 
the proportion of patients with diabetes that is seen within the HES. A very approximate estimate of $10 \%$ might be obtained from the Department of Health screening data, as 2192772 people were offered DR screening and 224711 were excluded from screening in the third quarter of $2012-13 ;^{41}$ the majority of those excluded would be expected to be those under the HES, but this figure may not be accurate as people may be excluded for other reasons. The total number of people with diabetes (diagnosed and undiagnosed) in the UK was estimated to be 3.8 million in 2011; according to this model, the number was predicted to rise to 5 million by $2025 .{ }^{4}$ Importantly, this projected increase is expected to be accompanied by an increase in the number of patients with diabetic complications, including DR. ${ }^{29}$

Prevalence figures are approximately three times higher in the NOD hospital data than wholepopulation estimates, reflecting the fact that more advanced grades of retinopathy are managed by the HES. The prevalence of any grade of DR was around $80 \%$ in our study (among patients undergoing a DR structured assessment), compared with $20-40 \%$ in population studies of patients with diabetes. PDR prevalence was around 20\% and CSMO prevalence was around $18 \%$, compared with $1-8 \%$ and $6 \%$, respectively, in the population. ${ }^{29-35}$ It is also difficult to make international comparisons for hospital DR prevalence data, as each country's health system has a different approach to thresholds for referral; hence, the proportion of patients with diabetes managed by the HES will be different from the proportion managed via primary care. The proportions of patients with DR managed by the UK HES should in theory be more consistent, as national screening programmes have been implemented to invite the whole UK population of patients with diabetes for screening and to refer to the HES only those with potentially sight-threatening retinopathy. However, some heterogeneity in referral rates between countries and regions may exist in the UK (as described above), though this may be reduced in future with the introduction of a common DESP pathway.

In conclusion, we have used the NOD to generate prevalence data on the grades of retinopathy and maculopathy for a large cohort of patients with diabetes managed by the HES in the UK. This sample is large and is likely to be broadly representative of those patients seen in UK NHS ophthalmology departments. These results have important implications for patients and clinicians, as well as managers and commissioners, of diabetic eye services, particularly when planning anti-VEGF services for eyes with CIDMO.

\section{Summary}

What was known before

- The management of patients with diabetic retinopathy forms an important part of hospital eye service activity in the United Kingdom.

\section{What this study adds}

- The UK National Ophthalmology Database is capable of collating standardised electronic medical record data on diabetic retinopathy and maculopathy from many hospital trusts.

- This can be used to generate clinically relevant information that is representative of the UK hospital eye service.

- Of people assessed for diabetic retinopathy in the UK hospital eye service, almost $10 \%$ of eyes have centreinvolving diabetic macular oedema, and around $20 \%$ have proliferative disease.

\section{Conflict of interest}

RL Johnston is the medical director of Medisoft Limited, which developed the electronic medical record from which data were extracted for the first iteration of the National Ophthalmology Database, but no funding was provided by the company for this study. TDL Keenan has consulted for Alimera Sciences and received compensation.

\section{Acknowledgements}

The work of the National Ophthalmology Database received initial funding from the Department of Health via the NHS Diabetic Eye Screening Programme (formerly the English National Screening Programme for Diabetic Retinopathy) and is currently partly funded by The Royal College of Ophthalmologists. See appendix.

\section{References}

1 Bunce C, Wormald R. Causes of blind certifications in England and Wales: April 1999-March 2000. Eye 2008; 22: 905-911.

2 The Royal College of Ophthalmologists. Preferred practice guidance. Diabetic retinopathy screening (DRS) and the Ophthalmology clinic set up in England 2010.

3 The Royal College of Ophthalmologists. Ophthalmic services guidance. The delivery of diabetic eye care 2009 .

4 Diabetes UK. Diabetes in the UK 2012, Key statistics on diabetes, URL http:/ /www.diabetes.org.uk/Documents/ Reports/Diabetes-in-the-UK-2012.pdf.

5 Scanlon PH. The English national screening programme for sight-threatening diabetic retinopathy. J Med Screen 2008; 15: $1-4$. 
6 NHS Scotland. NHS Scotland National Diabetes Retinopathy Screening, URL http://www.ndrs.scot.nhs.uk.

7 Cardiff and Vale University Health Board. Diabetic Retinopathy Screening Service for Wales (DRSSW), URL http:/ / www.cardiffandvaleuhb.wales.nhs.uk/drssw.

8 Public Health Agency, Belfast: Northern Ireland Diabetic Retinopathy Screening Programme, URL http:// www.publichealth.hscni.net/publications/northernireland-diabetic-retinopathy-screening-programme.

9 The Royal College of Ophthalmologists, Commissioning and value for money. Current issues and opportunities-diabetic retinopathy, URL http:/ / www.rcophth.ac.uk/page.asp?section $=635$ \&sectionTitle $=$ Current + issues + and + opportunities $+\% 2 \mathrm{D}+$ Diabetic + Retinopathy.

10 European Medicines Agency, CHMP post-authorisation summary of positive opinion for Lucentis, URL http:// www.ema.europa.eu/docs/en_GB/document_library/ Summary_of_opinion/human/000715/WC500098335.pdf.

11 National Institute for Health and Clinical Excellence. Ranibizumab for the treatment of diabetic macular oedema (rapid review of TA237). TA274. London: National Institute for Health and Clinical Excellence 2013.

12 The Royal College of Ophthalmologists. The Do Once and Share Connecting for Health Initiative, URL http:/ / www.rcophth.ac.uk/ page.asp? section $=387 \&$ sectionTitle $=$ The + Do + Once + and + Share + 'Connecting + for + Health' + Initiative.

13 The Royal College of Ophthalmologists. The profession, URL http://www.rcophth.ac.uk/ page.asp? section $=137 \&$ sectionTitle $=$ The + Profession.

14 The Royal College of Ophthalmologists. National Ophthalmology Database, URL http://www.rcophth. ac.uk $/$ page.asp? section $=668 \&$ sectionTitle $=$ National + Ophthalmology + Database.

15 Narendran N, Jaycock P, Johnston RL, Taylor H, Adams M, Tole DM et al. The Cataract National Dataset electronic multicentre audit of 55,567 operations: risk stratification for posterior capsule rupture and vitreous loss. Eye 2009; 23: 31-37.

16 Sparrow JM, Taylor H, Qureshi K, Smith R, Johnston RL. UK EPR user group. The cataract national data set electronic multi-centre audit of 55,567 operations: case-mix adjusted surgeon's outcomes for posterior capsule rupture. Eye 2011; 25: 1010-1015.

17 Johnston RL, Taylor H, Smith R, Sparrow JM. The Cataract National Dataset electronic multi-centre audit of 55,567 operations: variation in posterior capsule rupture rates between surgeons. Eye 2010; 24: 888-893.

18 Knox Cartwright NE, Johnston RL, Jaycock PD, Tole DM, Sparrow JM. The Cataract National Dataset electronic multicentre audit of 55,567 operations: when should IOLMaster biometric measurements be rechecked? Eye 2010; 24: 894-900.

19 Jaycock P, Johnston RL, Taylor H, Adams M, Tole DM, Galloway P, Canning C, Sparrow JM. UK EPR user group. The Cataract National Dataset electronic multi-centre audit of 55,567 operations: updating benchmark standards of care in the United Kingdom and internationally. Eye 2009; 23: 38-49.

20 Early Treatment Diabetic Retinopathy Study Research Group. Fundus photographic risk factors for progression of diabetic retinopathy. ETDRS report number 12 . Ophthalmology 1991; 98: 823-833.
21 NHS Diabetic Eye Screening Programme. URL http:/ / www.retinalscreening.nhs.uk.

22 Peto T, Tadros C. Screening for diabetic retinopathy and diabetic macular edema in the United Kingdom. Curr Diab Rep 2012; 12(4): 338-345.

23 Looker HC, Nyangoma SO, Cromie D, Olson JA, Leese GP, Black $\mathrm{M}$ et al. Diabetic retinopathy at diagnosis of type 2 diabetes in Scotland. Diabetologia 2012; 55(9): 2335-2342.

24 Kostev K, Rathmann W. Diabetic retinopathy at diagnosis of type 2 diabetes in the UK: a database analysis. Diabetologia 2013; 56(1): 109-111.

25 Bailey CC, Sparrow JM, Grey RH, Cheng H. The National Diabetic Retinopathy Laser Treatment Audit. I. Maculopathy. Eye 1998; 12: 69-76.

26 Bailey CC, Sparrow JM, Grey RH, Cheng H. The National Diabetic Retinopathy Laser Treatment Audit. II. Proliferative retinopathy. Eye 1998; 12: 77-84.

27 Williams R, Airey M, Baxter H, Forrester J, Kennedy-Martin T, Girach A. Epidemiology of diabetic retinopathy and macular oedema: a systematic review. Eye 2004; 18: 963-983.

28 Ding J, Wong TY. Current epidemiology of diabetic retinopathy and diabetic macular edema. Curr Diab Rep 2012; 12: 346-354.

29 The Royal College of Ophthalmologists. Guidelines for diabetic retinopathy 2005.

30 Morgan CL, Currie CJ, Stott NC, Smithers M, Butler CC, Peters JR. The prevalence of multiple diabetes-related complications. Diabet Med 2000; 17: 146-151.

31 Prasad S, Kamath GG, Jones K, Clearkin LG, Phillips RP. Prevalence of blindness and visual impairment in a population of people with diabetes. Eye 2001; 15: 640-643.

32 Sparrow JM, McLeod BK, Smith TD, Birch MK, Rosenthal AR. The prevalence of retinopathy and maculopathy and their risk factors in the non-insulin treated diabetic patients of an English town. Eye 1993; 7: 158-163.

33 Younis N, Broadbent DM, Harding SP, Vora JR. Prevalence of diabetic eye disease in patients entering a systematic primary care-based eye screening programme. Diabet Med 2002; 19: 1014-1021.

34 McLeod BK, Thompson JR, Rosenthal AR. The prevalence of retinopathy in the insulin-requiring diabetic patients of an English town. Eye 1988; 2: 424-430.

35 Broadbent DM, Scott JA, Vora JP, Harding SP. Prevalence of diabetic eye disease in an inner city population: the Liverpool Diabetic Eye Study. Eye 1999; 13: 160-165.

36 Raymond NT, Varadhan L, Reynold DR, Bush K, Sankaranarayanan S, Bellary S et al. Higher prevalence of retinopathy in diabetic patients of South Asian ethnicity compared with white Europeans in the community: a cross-sectional study. Diabetes Care 2009; 32: 410-415.

37 Yau JW, Rogers SL, Kawasaki R, Lamoureux EL, Kowalski JW, Bek T et al. Global prevalence and major risk factors of diabetic retinopathy. Diabetes Care 2012; 35: 556-564.

38 Minassian D, Reidy A. Future Sight Loss UK (2): Future Sight Loss in the Decade 2010 to 2020: An Epidemiological and Economic Model. Royal National Institute of Blind People-UK Vision Strategy: London, UK, 2009.

39 Klein R, Klein BE, Moss SE, Davis MD, DeMets DL. The Wisconsin epidemiologic study of diabetic retinopathy. IV. Diabetic macular edema. Ophthalmology 1984; 91: 1464-1474.

40 Yamamoto T, Iimuro S, Ohashi Y, Sone H, Yamashita H, Ito H. Japanese Elderly Intervention Trial Research Group. Prevalence and risk factors for diabetic maculopathy, and its 
relationship to diabetic retinopathy in elderly Japanese patients with type 2 diabetes mellitus. Geriatr Gerontol Int 2012; 12: 134-140.

41 Department of Health. Number of patients with diabetes receiving/offered screening for the early detection of diabetic retinopathy. Department of Health 2013: London, URL http:/ / transparency.dh.gov.uk/?p=20253.

\section{Appendix}

Appendix Centres contributing to the NOD are listed below

Centres for which DR structured assessment data were available

Aintree Hospitals NHS Trust; Barking, Havering, and Redbridge University Hospitals NHS Trust; Bedford Hospital NHS Trust; Bradford Teaching Hospitals NHS Foundation Trust; Calderdale and Huddersfield NHS Foundation Trust; Cambridge University Hospitals NHS Foundation Trust; Gloucestershire Hospitals NHS Foundation Trust; Epsom and St Helier University Hospitals NHS Trust; Frimley Park Hospital NHS
Foundation Trust; Leeds Teaching Hospitals NHS Trust; Mid Cheshire Hospitals NHS Foundation Trust; Mid Yorkshire Hospitals NHS Trust; Royal Berkshire NHS Foundation Trust; Royal United Hospital Bath NHS Trust; South London Health-care NHS Trust; University Hospitals Bristol NHS Foundation Trust; Winchester and Eastleigh Health-care NHS Trust; Wirral University Teaching Hospital NHS Foundation Trust.

\section{Centres for which DR structured assessment data were unavailable}

Dumfries and Galloway Community Health NHS Trust; Grampian Health-care NHS Trust; King's College Hospital NHS Trust; Nottingham University Hospital NHS Trust; Norfolk and Norwich University Hospitals NHS Foundation Trust; North Devon health-care NHS Trust; Peterborough and Stamford Hospitals NHS Foundation Trust; Portsmouth Hospitals NHS Trust; South Warwickshire NHS Foundation Trust; The Hillingdon Hospital NHS Trust; University Hospital Birmingham NHS Foundation Trust; University Hospital Of North Staffordshire NHS Trust. 